\section{BIRTH -SIZE DISTRIBUTION IN 4050 INFANTS TO MOTHERS WITH DIABETES TYPE 1. A NATION-WIDE STUDY.}

M. Persson ${ }^{1}$, D. Pasupathy², U. Hanson³, M. Norman ${ }^{4}$

${ }^{1}$ Department of Clinical Science, Intervention and Technology, Karolinska Insitutet, Stockholm, Sweden, ${ }^{2}$ Academic Department of Women's Health, Kings College, London, UK, ${ }^{3}$ Department of Woman and Child Health, Uppsala University, Uppsala, ${ }^{4}$ Department of Clinical Science, Intervention and Technology, Karolinska Institutet and University Hospital, Stockholm, Sweden

Objective: In spite of improved metabolic control during pregnancy, mothers with insulin-dependent diabetes mellitus (IDDM) are still at risk of delivering macrosomic infants. ${ }^{1}$ The aim of this study was to further characterize birth-size distribution in infants to IDDM mothers.

Methods: Population-based cohort study of infants ( $n=4,050 ; 2,054$ boys)born to IDDM mothers in Sweden between1998-2007 with a gestational age (GA) of 28-42 weeks.Birth weight (BW) and birth length $(B L)$ were retrieved from the Medical Birth Registry and expressed as SD-scores for healthy infants. Fatness was defined as BWSDS-BLSDS > +2 , and leanness as BWSDS-BLSDS $<-2 .{ }^{2}$ Values are mean (SD).

Results: Mean BW was 3744(749) g and GA 37.6(2.1)wks. Mean BWSDS for IDDM-infants was 1.28(1.48) and BLSDS 0.70(1.24).Both SDS were normally distributed. The $90^{\text {th }}$ percentile for BWSDS was 3.21 and the $97.5^{\text {th }}$ percentile was 4.41. Fatness was seen in $6.3 \%$ and leanness in $0.44 \%$ of the IDDM-infants.Term infants had higher BWSDS (mean1.31 vs 0.99, p<0.001), higher BLSDS (mean 0.73 vs $0.53, p<0.001)$ and higher proportion of fatness $(6.7$ vs $3.7 \%, p<0.01)$ than preterm IDDM infants, whereas leanness was similar in the two groups. Girls had higher BWSDS (1.36 vs $1.19, \mathrm{p}<$ 0.001 ) and higher BLSDS (0.76 vs $0.65, p<0.01$ ) than boys, whereas fatness/leanness was equally distributed among sexes.

Conclusion: Distributions for both $\mathrm{BW}$ and $\mathrm{BL}$ are significantly right-shifted in infants to IDDM mothers.A larger shift in BW- compared to BLdistribution indicates disproportional fetal growth. Further studies on causes and consequences of this early growth-deviation are warranted.

\section{References:}

1. Diabetes Care 2009;32:2005-9

2. Acta Paediatr $1993 ; 82: 333-9$

56

\section{CARDIOPULMONARY RESUSCITATION BY CHEST COMPRESSIONS VERSUS VENTILATION PLUS CHEST COMPRESSIONS IN A PEDIATRIC ASPHYXIAL CARDIAC ARREST ANIMAL MODEL}

M. Botran, J. Urbano, M.J. Solana, Y. Ballestero, J. López-Herce, D. Vinciguerra

Pediatric Intensive Care Service, Hospital General Universitario Gregorio Marañón, Madrid, Spain

Background and aims: To compare survival, ventilation and hemodynamic variables achieved with chest compressions or ventilation plus chest compressions in a pediatric animal model of cardiac arrest.

Design and methods: Experimental study on 30 infant pigs with asphyxial cardiac arrest, which were randomized into 2 groups of cardiopulmonary resuscitation (CPR): Group 1 (16 pigs) : chest compressions plus non coordinated ventilation with mask and a mechanical ventilator and FiO2 $21 \%$ (VC). Group 2 (14 pigs): only chest compressions (CC). After 9 minutes advanced resuscitation continued the same in both groups until complete 30 minutes.

Results: 3 animals (18.8\%) in group VC recovered spontaneous circulation while only $1(7.1 \%)$ in group $C C$ did it $(p=0.351)$. None of the groups achieved acceptable oxygenation and ventilation during the early CPR. Nevertheless both oxygenation and ventilation were significantly better in group VC than in group CC after 9 minutes (PaO2: 26 mmHg vs 19 $\mathrm{mmHg}, \mathrm{p}=0.008$; PaCO2: $84 \mathrm{mmHg}$ vs $101 \mathrm{mmHg}$, $p=0.05)$. Cerebral saturation was also higher in group VC $(61 \%)$ than in group CC $(30 \%), p=0.006$. In addition there were no significantly differences in arterial $\mathrm{pH}$ nor in mean arterial pressure.

\section{Conclusions:}

1-None of the early CPR protocol achieves adequate oxygenation and ventilation in this model of asphyxial cardiac arrest.

2- Chest compressions plus ventilation get better oxygenation and ventilation with no negative effect on the hemodynamic situation. 